\title{
Why Does the North Brazil Current Regularly Shed Rings but the Brazil Current Does Not?
}

\author{
VOLODYMYR ZHARKOV AND DORON NOF* \\ Geophysical Fluid Dynamics Institute, The Florida State University, Tallahassee, Florida
}

(Manuscript received 20 February 2009, in final form 24 June 2009)

\begin{abstract}
Both the North Brazil Current (NBC) and the Brazil Current (BC) are western boundary currents (WBCs) that separate from the western Atlantic coast. The NBC retroflects and sheds several rings per year (at the retroflection region), whereas the $\mathrm{BC}$ rarely sheds rings near its separation point. Traditionally, the difference between these two WBCs has been attributed to the Malvinas Current (MC), whose momentum flux opposes the poleward momentum flux of the $\mathrm{BC}$, thus preventing rings shedding at the point where the current leaves the coast. Even in the absence of the MC, rings from the separating BC would have never been regularly generated because of the relatively large slant of the coastline relative to the zonal direction.

Using the recently proposed theory of Zharkov and Nof, it is demonstrated that the large inclination of the coastline between $20^{\circ}$ and $45^{\circ} \mathrm{S}$ (approximately $50^{\circ}$ ) lies within the regime that does not allow the BC a continuous shedding of rings. In contrast, the inclination of the coastline between $5^{\circ}$ and $8^{\circ} \mathrm{N}$ is sufficiently small to allow the NBC a continuous and smooth shedding of rings. The importance of the coastline in clination comes about through a ring $\beta$-induced westward propagation rate. In the small inclination case, the alongshore migration is fast, allowing the newly formed rings to quickly escape from their generation zone (i.e., before they are recaptured by the newly born rings generated behind).

In contrast, in the high inclination case, the alongshore speed is so small that the rings spend a long time in the generation area and, consequently, are usually recaptured by the new rings generated just behind them. The authors argue, paradoxically, that the rings occasionally shed by the $\mathrm{BC}$ are probably due to the $\mathrm{MC}$ that advects the rings away from the generation area, preventing their recapture by the current behind them. Although no new analytical solutions are presented, the authors elaborate on the application of the recapturing condition to the NBC and BC and show new numerical simulations for both the NBC and the BC.
\end{abstract}

\section{Introduction}

The generation of rings is a fascinating process. The first rings that were examined in detail were the open ocean rings, such as the cold-core and warm-core Gulf Stream rings and Kuroshio rings, which were studied extensively in the 1970s and 1980s. Their formation has been correctly attributed to the instability of free zonal jets on a $\beta$ plane (see, e.g., Robinson 1983). Because there is open water on both sides of the jets, the rings are free to meander and snake around. Under some con-

* Additional affiliation: Department of Oceanography, The Florida State University, Tallahassee, Florida.

Corresponding author address: Doron Nof, The Florida State University, 419 OSB, 117 N. Woodward Ave., Tallahassee, FL 32306.

E-mail: nof@ocean.fsu.edu ditions, the meanders grow exponentially, close upon themselves, and pinch off a closed loop with either cyclonic or anticyclonic circulation (depending on whether the meander is convex or concave, looking northward). Note that by "instability," we refer to the classical definition, that is, the breakup of a known steady solution, not a mere transfer of energy from the mean flow to the eddy field - a definition sometimes used by numerical modelers.

At times, western boundary currents that are still in contact with the wall on the west also generate rings. This happens just as they separate from the coast and turn offshore to become free jets. Examples are the North Brazil Current (NBC), the East Australian Current (EAC), and the Loop Current. [Agulhas rings are not included in this category because of the low-latitude termination of the South African continent; see, e.g., Lutjeharms (2006).] The separations of the NBC and Brazil Current (BC) are shown in Figs. 1 and 2. In both 


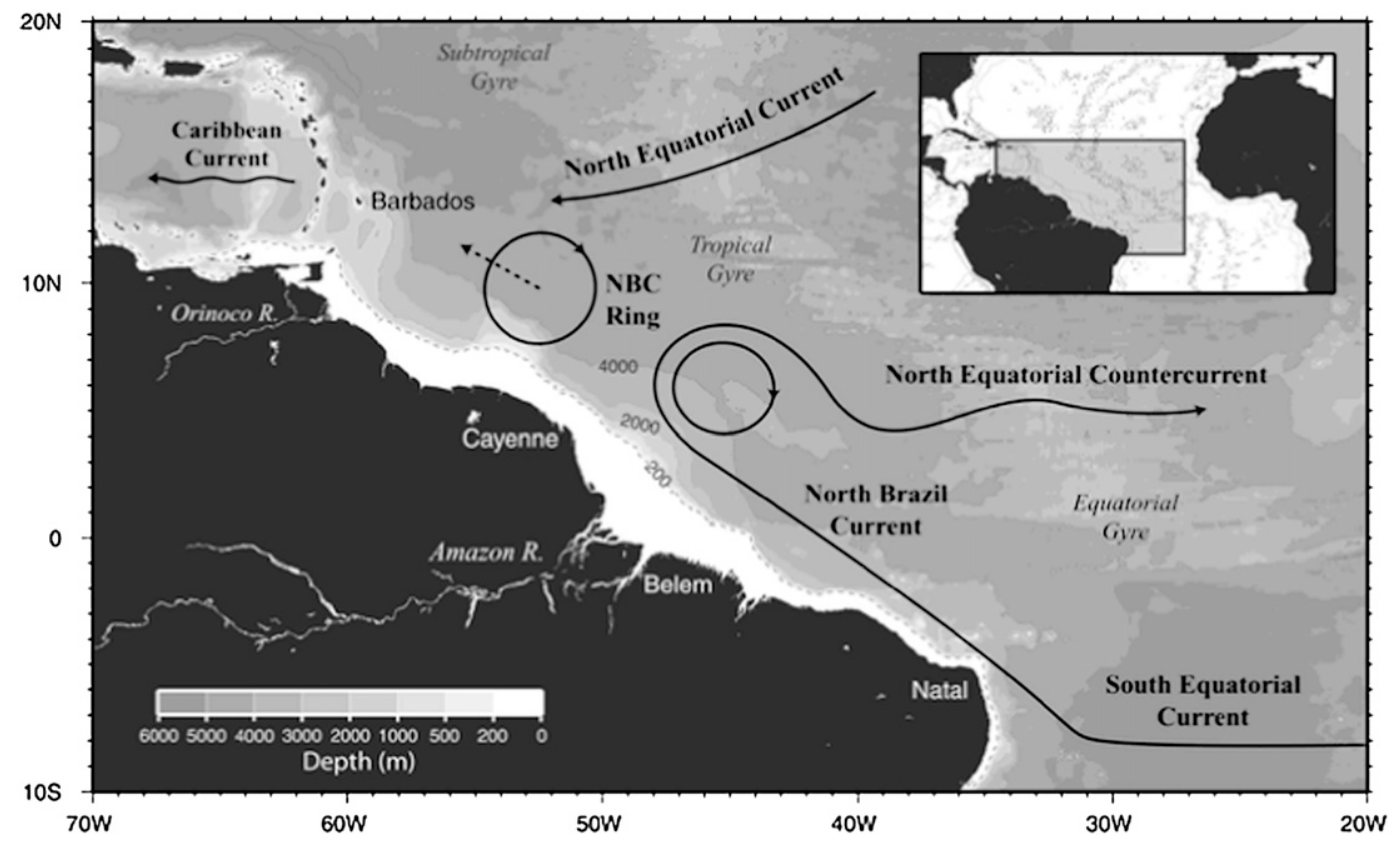

FIG. 1. Cartoon depicting the major upper-ocean circulation features in the western tropical Atlantic. The South Equatorial Current (SEC), North Brazil currnet (NBC), and North Equatorial countercurrent (NECC) close the wind-driven equatorial gyre. NBC rings provide a mechanism for mass and tracer transport from this gyre through the tropical gyre and into the southern extent of the North Atlantic subtropical gyre. Adapted from Fratantoni and Glickson (2002).

of these cases, the wall bounds the jets on the west, so they cannot freely meander while they are still attached to it, implying that a process different than the aforementioned classical instability must be in play. One process that has been suggested for the NBC is still related to instability-meanders are generated offshore and then propagate upstream (i.e., westward) to the separation or retroflection area (see, e.g., da Silveira et al. 1999; Jochum and Malanotte-Rizzoli 2003).

A different mechanism, related to an imbalance of alongshore momentum flux, was suggested by Pichevin and Nof (1996) and Pichevin et al. (1999). In their scenario, rings must be generated so as to compensate for the alongshore flow force, which is left unbalanced once the jets turn offshore. Supposedly, this idea is applicable to the NBC but not the $\mathrm{BC}$ because the opposing Malvinas Current $(\mathrm{MC}$ ) provides a balance to the otherwise unbalanced momentum flux (see, e.g., Nof 1996).

Recently, Zharkov and Nof (2008a,b, hereafter ZNa and $\mathrm{ZNb}$ ) suggested that this momentum flux mechanism has a critical dependency on the inclination of the coastline relative to east. When the western boundary is nearly meridional, no rings are generated because the westward drift is too slow to allow the rings to escape from the ring generation region. Namely, rings are generated but they are recaptured by the newly born rings generated immediately behind them. By contrast, when the western boundary is nearly zonal, the westward speed is high, allowing the rings to escape from their generation zone before they are recaptured. In between the two, there is a critical angle above which no rings are generated, for example, the $\mathrm{BC}$, and below which rings are regularly shed (NBC).

This paper is organized as follows. In section 2 we briefly review the not-so-trivial theoretical analysis of $\mathrm{ZNa}$ (sections $2 \mathrm{a}-2 \mathrm{c}$ ) and $\mathrm{ZNb}$ (section $2 \mathrm{~d}$ ), and in section 3 we present their application to the NBC and BC. In section 4 , we show the results of new numerical calculations for the ring shedding process under conditions similar to these of the BC and NBC. Section 5 is devoted to an estimation of outgoing fluxes of retroflected $\mathrm{BC}$ and NBC. In section 6, we summarize and discuss our results.

\section{Theory}

In this section, the theory originally presented in $\mathrm{ZNa}, \mathrm{b}$ for the Southern Hemisphere is briefly presented for the Northern Hemisphere. Although the final system of equations for volume flux and momentum flux (sections $2 \mathrm{~b}$ and $2 \mathrm{c}$ ) are the same as in $\mathrm{ZNa}$, some interstitial formulas in our present analysis will differ from those in $\mathrm{ZNa}$. The most important section is section $2 \mathrm{~d}$, where we will define and discuss the upper and lower boundaries of the ring size. 


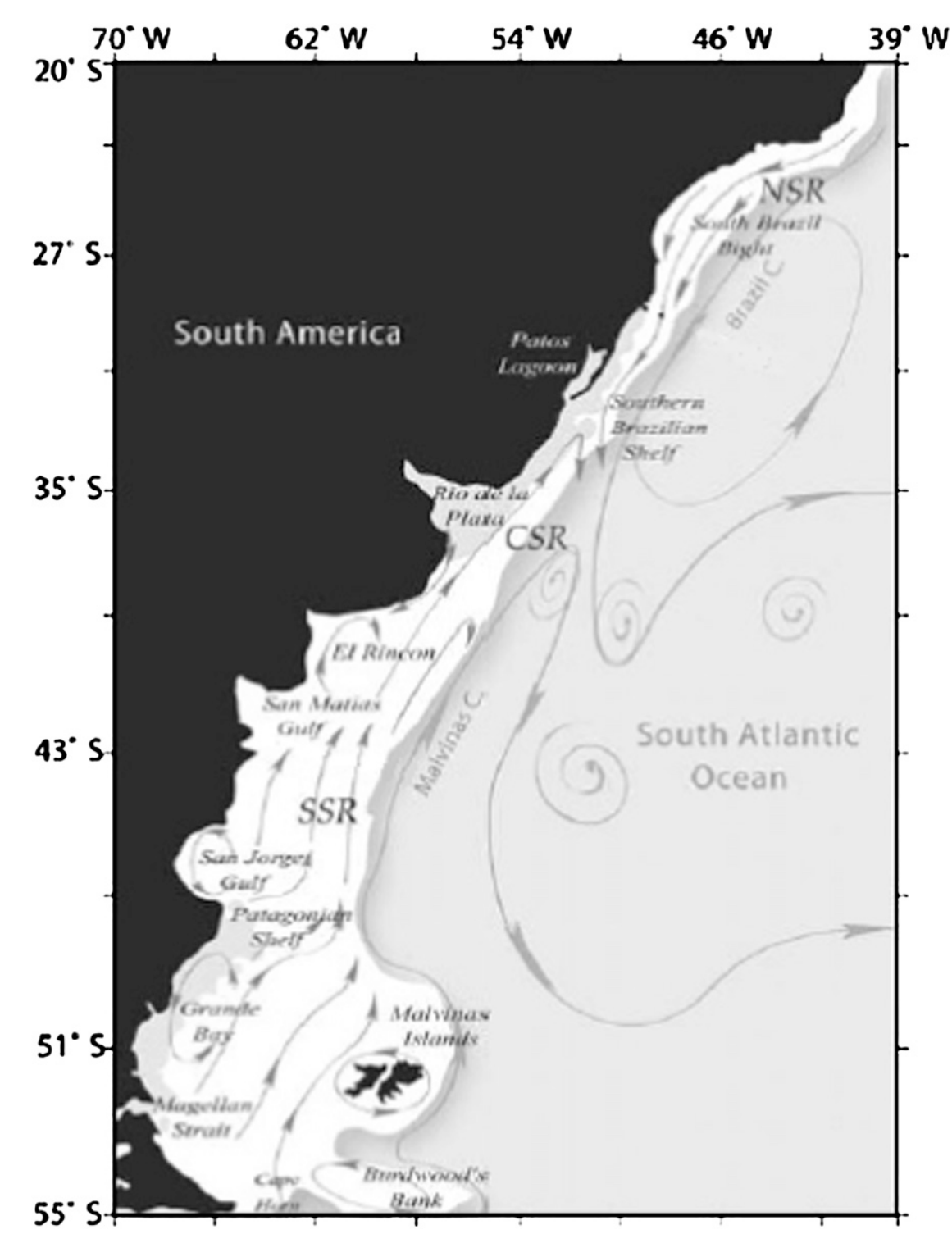

FIG. 2. Map showing the Brazil-Malvinas confluence. Heavy gray lines indicate the schematic path of the offshore boundary currents, the Brazil and Malvinas Currents. Adapted from Palma et al. (2008).

\section{a. Formulation}

Following $\mathrm{ZNa}$, we consider a boundary current (with density $\rho$ ) embedded in an infinitely deep, stagnant lower layer with density $(\rho+\Delta \rho)$ but, as mentioned, we now consider the complimentary Northern Hemisphere problem. The current is flowing along a rectilinear wall and retroflects at some point (Fig. 3). The wall slant relative to the zonal direction $(\gamma)$ varies between zero for a zonal coastline and $90^{\circ}$ for a meridional coastline. We shall use an offshore and an alongshore coordinate $\operatorname{system}(\xi, \eta)$ moving with the developing basic eddy (BE). The transformation formulas are $\xi=x \cos \gamma-y \sin \gamma, \eta=x \sin \gamma+$ $y \cos \gamma$. The orbital velocity of the $\mathrm{BE}$ is $-\alpha f_{0} r / 2$, where $\alpha$ is the nondimensional vorticity coefficient and $f_{0}$ is the mean local value of the Coriolis parameter. (Variables are conventionally defined in both text and the appendix.)

Using an expansion with the small parameter, $\varepsilon=$ $\left(\beta R_{d} / f_{0}\right)$ in which $R_{d}$ is the Rossby radius, we obtain the volume conservation equation

$$
\frac{d}{d t} \iint_{s} h d \xi d \eta=Q-q
$$

where $h$ is thickness of the upper layer inside the BE and $Q$ and $q$ are the mass fluxes of the incoming and outgoing currents. The long-wall momentum equation that we will use is 


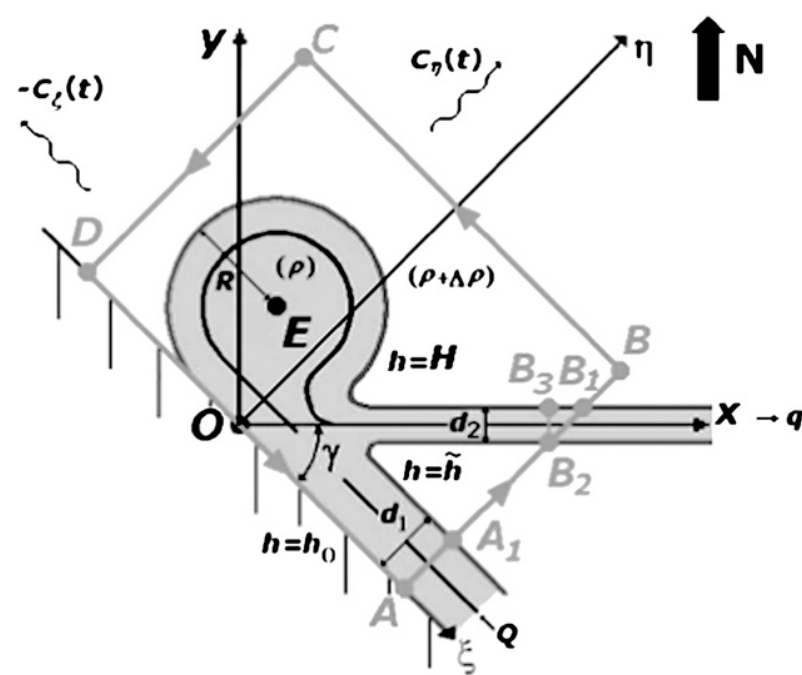

FIG. 3. A schematic diagram of the model under study: $E$ is the center of the base eddy (BE). In the tilted coordinate system $\xi$ is directed along the coastline and $\eta$ is directed normal to the coastline. Incoming flux $Q$ flows along the wall, whereas outgoing flux $q$ is directed to the east. The wavyarrow indicates the migration of the $\mathrm{BE}$; it results from both the eddy growth, which forces the eddy away from the wall, and from $\beta$, which forces the eddy along the wall. We shall see that the migration $C_{\eta}(t)$ is primarily due to the growth, whereas $C_{\xi}(t)$ is primarily due to $\beta$. The thick gray line (with arrows) indicates the integration path $A B C D A ; \tilde{h}$ is the upper-layer thickness of the stagnant region wedged in between the upstream and retroflecting current, and $H$ is the offshore thickness. The segment $B_{2} B_{3}$ is involved in the expressions for $\gamma$ (see text).

$$
\begin{aligned}
& -\frac{\partial^{2} \psi}{\partial \eta \partial t}+h \frac{\partial C_{\xi}}{\partial t}+\frac{\partial\left(h u^{* 2}\right)}{\partial \xi}+\frac{\partial\left(h u^{*} v^{*}\right)}{\partial \eta}-f \frac{\partial \psi}{\partial \xi} \\
& -f h C_{\eta}+\frac{g^{\prime}}{2} \frac{\partial h^{2}}{\partial \xi}=0,
\end{aligned}
$$

where $u^{*}$ and $v^{*}$ are the velocities in the $\xi$ and $\eta$ directions, $C_{\xi}$ and $C_{\eta}$ the $\mathrm{BE}$ propagation rates, $\psi$ the streamfunction (defined by $\partial \psi / \partial \eta=-h u^{*}, \partial \psi / \partial \xi=$ $\left.h v^{*}\right), g^{\prime}$ the reduced gravity, and the Coriolis parameter $f$ is defined as $f=f_{0}-\beta \xi \sin \gamma$.

\section{b. Momentum fluxes}

Next, we integrate (2.2) over the area bounded by the thick gray arrowed line shown in Fig. 3. Using the Stokes theorem and taking into account that $\partial f / \partial \xi=-\beta \sin \gamma$, one obtains

$$
\begin{gathered}
\oint_{\varphi}\left[\frac{\partial \psi}{\partial t}-h u^{*} v^{*}\right] d \xi+\oint_{\varphi}\left[h u^{*^{2}}+\frac{g^{\prime} h^{2}}{2}-f \psi\right] d \eta \\
+\iint_{S}\left[h \frac{\partial C_{\xi}}{\partial t}-\beta|\psi| \sin \gamma-f h C_{\eta}\right] d \xi d \eta=0 .
\end{gathered}
$$

Keeping only the main contributions, we rewrite (2.3) in the form:

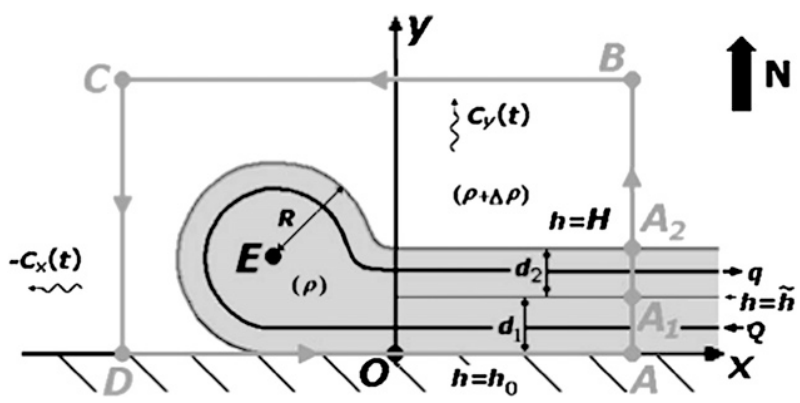

FIG. 4. A schematic diagram for the special case of a zonal coastline $(\gamma=0)$. Here, we use the coordinate system $(x, y)$ instead of $\xi$ and $\eta$. The fluxes $Q$ and $q$ are separated by the streamline at $x>0$ and $y=d_{1}$ so that the combined segment $A A_{2}$ serves as an analog of both $A A_{1}$ and $B_{2} B_{1}$, shown in Fig. 3. The wavy arrows show the migration of the eddy (northward on account of the $\mathrm{BE}$ growth and westward due to $\beta$ ).

$$
F_{1}+F_{2} \cos \gamma-F_{3}-F_{4} \sin \gamma=0,
$$

where

$$
\begin{aligned}
& F_{1}=\int_{A}^{A_{1}} h u^{* 2} d \eta ; \quad F_{2}=\int_{B_{2}}^{B_{3}} h u^{* 2} d l \\
& F_{3}=C_{\eta} \iint_{S} f h d \xi d \eta ; \quad F_{4}=\beta \iint_{S}|\psi| d \xi d \eta .
\end{aligned}
$$

Equation (2.4) is a balance of (i) the jetlike force of the incoming current, (ii) the force of the retroflected current downstream, (iii) the integrated Coriolis force resulting from the growth of the $\mathrm{BE}$, and (iv) an equatorward $\beta$ force resulting from the clockwise rotation of eddy particles within the eddy. Because the BE is off the wall, we do not expect the so-called image effect to be important and neither do we expect leakages of the kind discussed by Nof (1999) to be important.

For simplicity, we shall first consider the limiting $\gamma \rightarrow 0$ case (Fig. 4), introduce the new notations, and return to the original coordinate system $(x, y)$. The integration segment for $F_{2}$ is now $A_{1} A_{2}$ so that we have

$$
\begin{aligned}
& F_{1}=\int_{0}^{d_{1}} h u^{2} d y, \quad F_{2}=\int_{d_{1}}^{d_{1}+d_{2}} h u^{2} d y, \\
& F_{4}=\beta \iint_{S}|\psi| d x d y,
\end{aligned}
$$

where the expression for $F_{3}$ remains the same as before, and $d_{1}$ and $d_{2}$ are the widths of the incoming and retroflected currents.

Assuming that the velocity of the incoming and retroflected currents linearly depends on $y$ and satisfying the boundary conditions associated with (i) conservation of the Bernoulli integral along the boundaries of the currents and (ii) continuity of velocities between currents and the $\mathrm{BE}$, we obtain for the incoming current 
$u=\frac{\alpha f_{0}}{2}(y-R), \quad 0 \leq y \leq d_{1}, \quad x \geq 0$

and for the retroflected current

$u=\frac{\alpha f_{0}}{2}\left(y+R-d_{1}-d_{2}\right), \quad d_{1}<y \leq d_{1}+d_{2}$.

Both currents are geostrophic so that

$$
\frac{\partial h}{\partial y} \approx-\frac{f_{0}}{g} u(y),
$$

which, upon combining with (2.7) and taking into account that $h$ should be continuous across the streamline separating the incoming and retroflected currents $\left(y=d_{1}\right)$, gives

$$
h=\frac{\alpha f_{0}^{2}}{4 g^{\prime}} y(2 R-y)+h_{0}, \quad 0 \leq y \leq d_{1}
$$

and

$$
\begin{aligned}
h= & \frac{\alpha f_{0}^{2}}{4 g^{\prime}}\left\{y\left[2\left(d_{1}+d_{2}-R\right)-y\right]\right. \\
& \left.+2 d_{1}\left(2 R-d_{1}-d_{2}\right)\right\}+h_{0}, \quad d_{1}<y \leq d_{1}+d_{2} .
\end{aligned}
$$

Next, we invoke the volume fluxes relationships

$$
Q=-\int_{0}^{d_{1}} h u d y, \quad q=\int_{d_{1}}^{d_{1}+d_{2}} h u d y,
$$

which, using (2.7) and (2.9), gives

$$
d_{1,2}=R-\delta_{1,2}
$$

where

$\delta_{1}=\left[R^{2}-\frac{4 g^{\prime}}{\alpha f_{0}^{2}}\left(\tilde{h}-h_{0}\right)\right]^{1 / 2}, \quad \delta_{2}=\left[R^{2}-\frac{4 g^{\prime}}{\alpha f_{0}^{2}}(\tilde{h}-H)\right]^{1 / 2}$

and

$$
\tilde{h}=\left[h_{0}^{2}+\frac{2 f_{0} Q}{g^{\prime}}\right]^{1 / 2}, \quad H=\left[h_{0}^{2}+\frac{2 f_{0}(Q-q)}{g^{\prime}}\right]^{1 / 2} .
$$

With the aid of the above we now continue with the balance of forces in the special case of $\gamma \rightarrow 0$. To obtain the expressions for $F_{1}$ and $F_{2}$, we substitute (2.7), (2.9), and (2.11)-(2.13) into (2.6). After some algebra, we find

$$
F_{1}=\frac{\alpha^{3} f_{0}^{4}}{240 g^{\prime}}\left(2 R^{5}-5 \delta_{1}^{3} R^{2}+3 \delta_{1}^{5}\right)+\frac{\alpha^{2} f_{0}^{2} h_{0}}{12}\left(R^{3}-\delta_{1}^{3}\right)
$$

and

$$
\begin{aligned}
F_{2}= & \frac{\alpha^{3} f_{0}^{4}}{240 g^{\prime}}\left[2 R^{5}-5\left(\delta_{1}^{2}-\delta_{2}^{2}\right) R^{3}-5 \delta_{2}^{3} R^{2}+5 \delta_{1}^{2} \delta_{2}^{3}-2 \delta_{2}^{5}\right] \\
& +\frac{\alpha^{2} f_{0}^{2} h_{0}}{12}\left(R^{3}-\delta_{2}^{3}\right) .
\end{aligned}
$$

Assuming the $\mathrm{BE}$ to be circular, we obtain the leadingorder expressions for $F_{3}$ and $F_{4}$ by using the relationships

$$
h(r)=\frac{\alpha(2-\alpha) f_{0}^{2}}{8 g^{\prime}}\left(R^{2}-r^{2}\right)+H
$$

and

$$
V=\pi R^{2}\left[\frac{\alpha(2-\alpha) f_{0}^{2} R^{2}}{16 g^{\prime}}+H\right]
$$

Under the assumption $C_{\eta}=d R / d t$, we get

$$
F_{3}=\pi R^{2} f_{0}\left[\frac{\alpha(2-\alpha) f_{0}^{2} R^{2}}{16 g^{\prime}}+H\right] \frac{d R}{d t} .
$$

Similarly, we obtain

$$
F_{4}=\frac{\pi \alpha \beta f_{0} R^{4}}{8}\left[\frac{\alpha(2-\alpha) f_{0}^{2} R^{2}}{24 g^{\prime}}+H\right] .
$$

This completes our presentation of the forces calculation in the limiting case of a zonal wall $(\gamma \rightarrow 0)$. Returning now to the most general case of nonzonal wall $(\gamma \neq 0)$, we finally find with (2.4), (2.14), (2.15), (2.17), and (2.18) a nonlinear equation for $d R / d t$ representing the momentum flux along the coast:

$$
\begin{aligned}
& \frac{\left(\alpha f_{0}\right)^{3}}{240}\left[2(1+\cos \gamma) R^{5}-5 \cos \gamma\left(\delta_{1}^{2}-\delta_{2}^{2}\right) R^{3}-5\left(\delta_{1}^{3}+\delta_{2}^{3} \cos \gamma\right) R^{2}+\left(3 \delta_{1}^{5}+5 \delta_{1}^{2} \delta_{2}^{3} \cos \gamma-2 \delta_{2}^{5} \cos \gamma\right)\right] \\
& +\frac{\alpha^{2} f_{0} g^{\prime} h_{0}}{12}\left[(1+\cos \gamma) R^{3}-\left(\delta_{1}^{3}+\delta_{2}^{3} \cos \gamma\right)\right]-\pi R^{2}\left[g^{\prime} H\left(\frac{d R}{d t}+\frac{\alpha \beta R^{2}}{8} \sin \gamma\right)\right. \\
& \left.\quad+\frac{\alpha(2-\alpha) f_{0}^{2} R^{2}}{16}\left(\frac{d R}{d t}+\frac{\alpha \beta R^{2}}{12} \sin \gamma\right)\right]=0 .
\end{aligned}
$$


c. Combining the mass conservation into the momentum flux

To close the system of differential equations, we use (2.1) and (2.16) to find approximately

$$
\begin{aligned}
Q-q= & \frac{\mu}{g^{\prime}}\left\{\frac{\alpha(2-\alpha) f_{0}^{2} R^{2}}{8}+f_{0} \mu+\left[\left(f_{0} \mu\right)^{2}\right.\right. \\
& \left.\left.+\frac{\alpha(2-\alpha) f_{0}^{3} R^{2} \mu}{4}+\left(g^{\prime} h_{0}\right)^{2}\right]^{1 / 2}\right\},
\end{aligned}
$$

where $\mu=2 \pi R d R / d t$. At this point, substitution of the mass conservation (2.20) into the geostrophic and circular conditions (2.12), (2.13), and the momentum Eq. (2.19) leads to our desired single differential equation (of the first order) for $R$ as a function of $t$ with the known parameters $Q, h_{0}, g^{\prime}, f_{0}, \alpha, \beta$, and $\gamma$. This equation is extremely cumbersome and, therefore, is not presented here in detail. Hereafter, it will be referred to as the "radius-time equation," and its solution will be discussed later. For the initial conditions at $t=0$, we take the initial radius $R_{i}$ of the $\mathrm{BE}$ to coincide with the width of incoming current $d_{1}$. Numerical solution of the aforementioned radius-time equation yields the functions $R=R(t)$, and $H=H(t)$, which will be discussed in the next subsection.

\section{d. "Lower" and "upper" ring radii and the critical coastline slant}

In the open ocean, $\beta$ forces nonlinear eddies westward (Nof 1983) at the speed

$$
C \approx-\beta\left[\frac{\alpha R^{2}}{12}+\frac{2 g^{\prime} H}{(2-\alpha) f_{0}^{2}}\right] .
$$

In the general case of a nonzonal wall, the velocity component along the wall is

$$
C_{\xi}=C \cos \gamma
$$

Given the downstream geometry (Fig. 5a), the generation period for each individual eddy is

$$
t_{f}=\frac{\left(2 R_{f}+d\right)}{\left|C_{\xi f}\right|}
$$

where $d$ is the distance between two consecutive eddies and the subscript $f$ denotes the "final" value. The "lower boundary" of the final eddy size $R_{f}$ is obtained using the
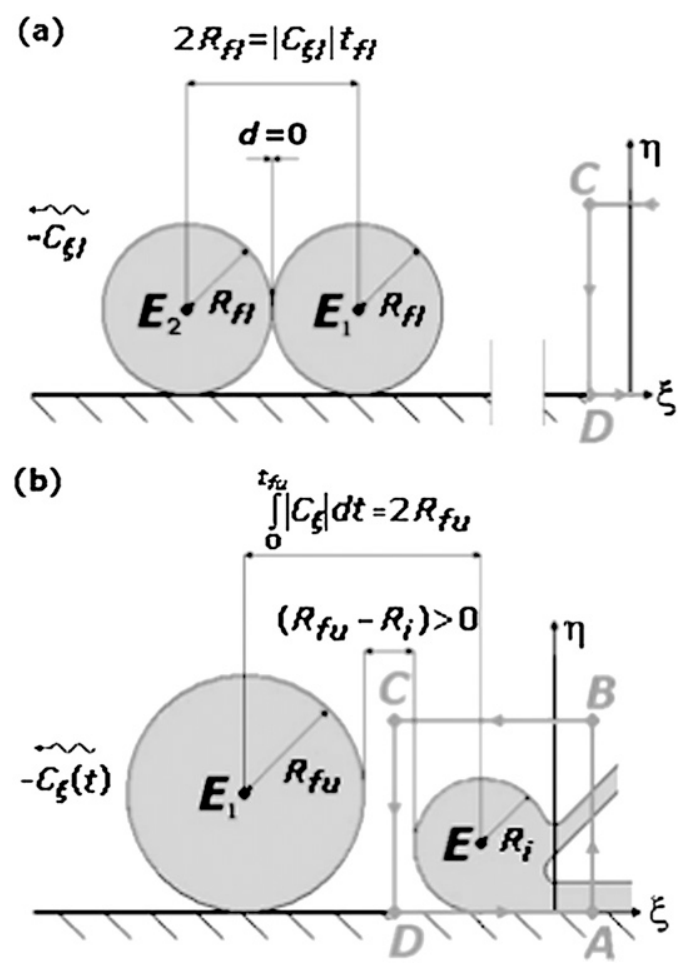

FIG. 5. Geometries associated with the lower and upper boundaries of the final eddy radius and detachment periods. (top) Two consecutive osculating eddies $(d=0)$ away from the retroflection area corresponding to the lower limit. Specifically, the lower limit on the detachment period $t_{f t}$ is obtained by a division of the doubledfinal-eddy radius $2 R_{f l}$ by the modulus of the final eddy migration rate. The segment $C D$ corresponds to the western boundary of the integration area (see Figs. 3 and 4). (bottom) The upper limit on the detachment period shows the already detached eddy (centered in $\left.E_{1}\right)$ migrating at the rate $-C_{\xi}(t)$ and an incipient BE centered in $E$. At the moment, $t_{f u}$, the distance between the two eddies is $\left(R_{f u}-R_{i}\right)$, which, as should be the case, is positive because the incipient $\mathrm{BE}$ is less developed. Therefore, our $A B C D$ contour encloses only the incipient BE.

so-called kissing condition used earlier by Pichevin and Nof (1996); that is, $d=0$, Fig. 5a. In that case, (2.21)(2.23) give

$$
t_{f l}=\frac{24(2-\alpha) f_{0}^{2} R_{f l}}{\beta\left[\alpha(2-\alpha) f_{0}^{2} R_{f l}^{2}+24 g^{\prime} H_{f l}\right] \cos \gamma} .
$$

This is the lower limit on the time required for the ring generation because the rings cannot overlap each other. Equivalently, eddies with $d>0$ will have a radius larger than that given by (2.24). Together with the radius-time equation, (2.24) is later solved (numerically) giving the radius as a function of time. We shall now leave this integration issue temporarily aside. 
Next, we derive the intricate and somewhat hard-tounderstand "upper boundary" $R_{f u}$ for the final BE size (i.e., the detachment size). Consider the configuration shown in Fig. 5b. During the generation period, the eddy is moving along the $\xi$ axis with velocity $C_{\xi}$, which is a function of time and is defined by (2.21) and (2.22). The displacement of the BE from its initial position during the generation period is $\int_{0}^{t_{f}}\left|C_{\xi}\right| d t$, where $C_{\xi}$ grows gradually as the eddy develops. We note that, when this displacement equals the final diameter of the eddy (i.e., $2 R_{f}$ ), then it must be detached because, at this point in time, it osculates the already-generated eddy farther downstream (whose radius must also be $R_{f}$ ).

Since the distance between the centers of the two consecutive eddies is at least the sum of their radii, we can place the segment of the integration contour surrounding the area of the BE between the two eddies (Fig. 5b), indicating that the formation of the second eddy is now in progress as the first one has already been fully developed and shed. In view of this, we write the condition of upper boundary in the form

$$
\int_{0}^{t_{f u}}\left|C_{\xi}\right| d t=2 R_{f u}
$$

which is an equation for $R_{f u}$. Our numerical simulations suggest that this limit assures that the growing time of the $\mathrm{BE}$ is short enough to allow it to escape from the generation area before it is captured by the newly born eddy generated behind it. In other words, when the generation time associated with (2.25) is longer than the migration time, then the $\mathrm{BE}$ will be recaptured by the new eddy being established upstream behind it. Alternatively, we can say that, if the eddy is larger than the radius corresponding to (2.25), then it occupies too much space and, hence, will be captured by the newly established eddy behind. It is important to realize at this point that eddies cannot just "push" each other ahead, because two eddies that bump into each other have a strong tendency to merge. This merging problem has been studied extensively in the 1980s and 1990s; for example, refer to Nof and Simon (1987) and the references given therein.

Physically, the upper boundary corresponds to the detachment of eddies during their formation (the generated ring must travel a distance at least equal to its own diameter), whereas the lower boundary corresponds to a condition for the formation of an eddy chain downstream (i.e., two consecutive rings can, at the most, touch each other but not overlap). Consequently, eddies can detach and propagate out of the retroflection area when the condition $R_{f l} \leq R_{f u}$ is satisfied, and we call this the subcritical range of the coastal slant. Otherwise, the

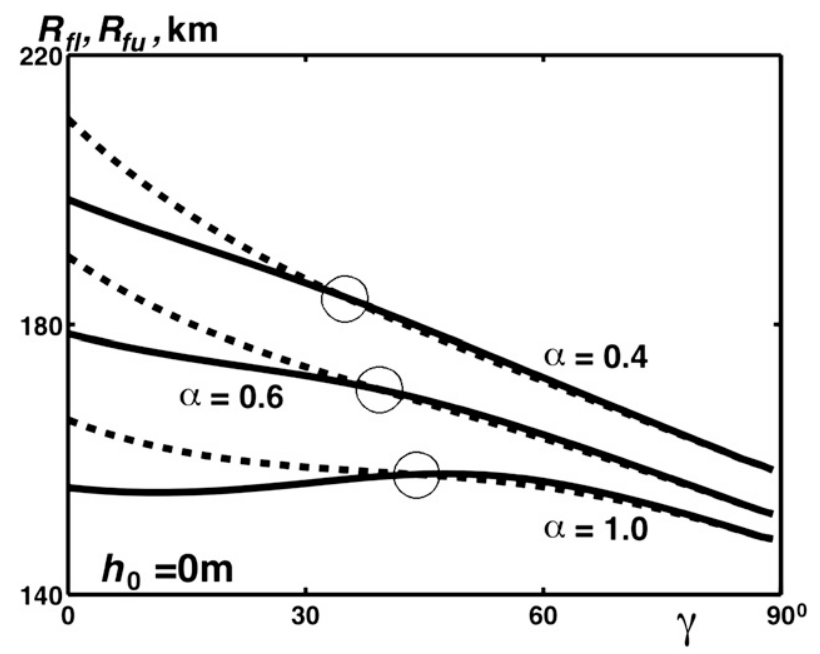

FIG. 6. Lower and upper boundaries of the detached ring radii vs the coastal slant (for the BC). The critical angle (circled) is their intersection because to the left of the intersection the upper limit is larger than the lower limit, as should be the case; however, to the right of the intersection the lower limit is larger than the upper limit, which is an impossible physical situation.

condition $R_{f l}>R_{f u}$ defines the supercritical range in which a systematic recapturing prevents the continuous formation of rings. We shall see later how these two important limits control the critical angle of the coastline.

\section{Application to the $\mathrm{BC}$ and $\mathrm{NBC}$}

In what follows we discuss the implication of the theory to the two cases under consideration. The tilt of coastline adjacent to the $\mathrm{BC}$ is about $50^{\circ}$ and $Q$ is about $20 \mathrm{~Sv}\left(\mathrm{~Sv} \equiv 10^{6} \mathrm{~m}^{3} \mathrm{~s}^{-1}\right)$ near the Brazil-Malvinas confluence zone (Olson et al. 1988; Peterson and Stramma 1991). In the theoretical calculations, $\gamma$ was varied between $0^{\circ}$ and $89^{\circ}$. The other parameters are the same as those used in $\mathrm{ZNb}, g^{\prime}=2 \times 10^{-2} \mathrm{~m} \mathrm{~s}^{-2}$ and $f_{0}=8.8 \times$ $10^{-5} \mathrm{~s}^{-1}$ (corresponding to $35^{\circ}$ latitude). We took 0 and $300 \mathrm{~m}$ for $h_{0}$ and $2.3 \times 10^{-11} \mathrm{~m}^{-1} \mathrm{~s}^{-1}$ in the (natural) theoretical calculations and $6 \times 10^{-11} \mathrm{~m}^{-1} \mathrm{~s}^{-1}$ for the stretched $\beta$, which we used in the numerics. In the theoretical calculations, $\alpha$ varied between 0.1 and 1.0.

Figure 6 shows the plots of the lower radius $R_{f l}$ (solid lines) and upper radius $R_{f u}$ (dashed lines) versus the coastline tilt $\gamma$ (for $h_{0}=0 \mathrm{~m}$ ). These solutions were obtained by solving the radius-time equation, discussed at the end of section $2 \mathrm{c}$, with either (2.24) or (2.25). The vorticity $\alpha$ again varied from 0.4 to 1.0 . The critical angle is the angle corresponding to the intersection of the solid and dashed curves for the same $\alpha$ (shown with circles in the figure). This is because, when the upper limit is 


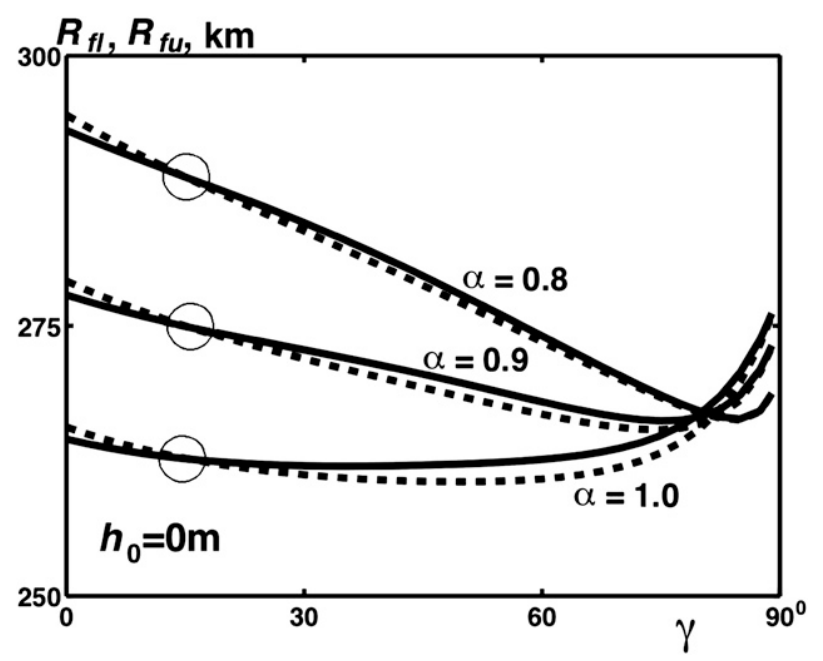

FIG. 7. As in Fig. 6 but for the NBC.

above the lower limit, rings are generated but they are not generated when it is the other way around (i.e., upper limit below the lower limit). We see that, when $h_{0}$ is zero, the critical slant changes from $33^{\circ}$ for $\alpha=0.4$ to $42^{\circ}$ for $\alpha=1.0$, implying that the tilt of the Brazilian coastline $\left(50^{\circ}\right)$ is inside the supercritical range of slants. This means that continuously detached rings are not expected. When we increase the thickness to $h_{0}=300 \mathrm{~m}$, the critical angle increased dramatically, and the tilt of the Brazilian coastline is closer to being critical. For $\alpha>$ 0.4 , it is even in the subcritical range. We should note here, however, that for retroflecting currents in subtropical regions, $\alpha$ is usually small, especially when the upper (disturbed) layer is relatively deep. According to Lentini et al. (2001), we can choose $R=110 \mathrm{~km}, f_{0}=$ $0.9 \times 10^{-4} \mathrm{~s}^{-1}$ and $u=55 \mathrm{~cm} \mathrm{~s}^{-1}$, giving $\alpha \approx 0.11$. Therefore, the formation of a detached eddies chain is unlikely even for relatively large $h_{0}$.

For the NBC we took $f_{0}=2 \times 10^{-5} \mathrm{~s}^{-1}, g^{\prime}=2 \times$ $10^{-2} \mathrm{~m} \mathrm{~s}^{-2}$, and $Q=35 \mathrm{~Sv}$ (Bourles et al. 1999; Johns et al. 1998; da Silveira et al. 1994; Schott et al. 1998). The plots for $R_{f l}$ and $R_{f u}$ versus $\gamma$ are shown in Fig. 7. By comparing Figs. 6 and 7, it is seen that the critical angle strongly decreases with decreasing Coriolis parameter. (Note, however, that for very small Coriolis parameter, the small parameter $\varepsilon=\beta R_{d} / f_{0}$ becomes large, invalidating the small $\varepsilon$ expansion.) For such a low-latitude, the critical angle changes from approximately $13^{\circ}$ to $15^{\circ}$ for large $\alpha$ varying from 1.0 to 0.8 . Note that such a large choice for $\alpha$ in our theoretical approach is justified here for two reasons. First, smaller $\alpha$ gives nonphysical, very large eddy radii. Second, for such a small Coriolis parameter, the "vorticity paradox" (appearing in the case of retroflection from a zonal wall, see $\mathrm{ZNa}$ ) is circumvented even for an angle as small as $1^{\circ}$ (compared to the $15^{\circ}$, which is the case for both the Agulhas Current and the BC). Moreover, even for a zonal wall and $\alpha=1$, the ratio of the mass flux going into the development of the $\mathrm{BE}$ and the total incoming mass flux does not surpass unity before the eddy detachment (see ZNa,b). We also note that, following Bischof et al. (2003), we can equally take $R=200 \mathrm{~km}, f_{0}=2 \times 10^{-5} \mathrm{~s}^{-1}$, and $u=1 \mathrm{~m} \mathrm{~s}^{-1}$, giving $\alpha \approx 0.5$. The theoretical calculations give again a critical slant of approximately $13^{\circ}$ (not shown in the figure). Hence, our theory confirms that NBC rings must be shed, at least when the retroflection protrudes westward to nearly $52.5^{\circ} \mathrm{W}$, and the eddies begin propagating near Paramaribo. We note here in passing that the most logical location for the NBC retroflection is $50^{\circ}-54^{\circ} \mathrm{W}$ (Lumpkin and Garzoli 2005). We shall return to this point later in section 5 .

\section{Numerical simulations}

For the numerical simulations, we used the same model as ZNa,b; namely, a modified version of the Bleck and Boudra (1986) reduced-gravity isopycnic model with a passive lower layer employing the Orlanski (1976) second-order radiation conditions for the open boundary. The downstream streamlines were not strongly disturbed when they crossed the boundary, suggesting that these open boundary conditions are satisfactory. In addition, changing the location of the boundary did not alter the behavior of the interior flow, which also indicates that the boundary conditions are appropriate.

We chose the initial potential vorticity (PV) in such a way that the starting value of $\alpha$ was $0.1,0.4$, and 1.0 . We note that frictional forces gradually altered the PV; consequently, $\alpha$ decreased during the experiments. The basin size was $3200 \times 1600 \mathrm{~km}^{2}$. For the conditions of the BC, the slanting continent was modeled by one fixed wall titled at an angle of $50^{\circ}$. Namely, the wall extended from the point $x=140 \mathrm{~km}, y=0$ to $x=1490 \mathrm{~km}, y=$ $1600 \mathrm{~km}$ and was taken to be slippery. The mass flux source was an open channel containing streamlines parallel to the wall for the incoming current and zonal for the outgoing flow (approximately at $y=1200 \mathrm{~km}$ ).

The experiments began by turning on the flow at $t=0$. As in our theoretical model, the initial velocity profile across the channel was linear, and the depth profile was parabolic. The numerical parameters of the model were a time step of $120 \mathrm{~s}$, a grid step of $20 \mathrm{~km}$, and a Laplacian viscosity coefficient of $\nu=700 \mathrm{~m}^{2} \mathrm{~s}^{-1}$. The reduced gravity was $g^{\prime}=2 \times 10^{-2} \mathrm{~m} \mathrm{~s}^{-2}$, the Coriolis parameter $f_{0}=8.8 \times 10^{-5} \mathrm{~s}^{-1}$, and the prescribed flow $Q=20 \mathrm{~Sv}$. To make the development of the BE faster and less sensitive to the distorting effect of viscosity, we artificially magnified $\beta$ to $6 \times 10^{-11} \mathrm{~m}^{-1} \mathrm{~s}^{-1}$. (We note here 

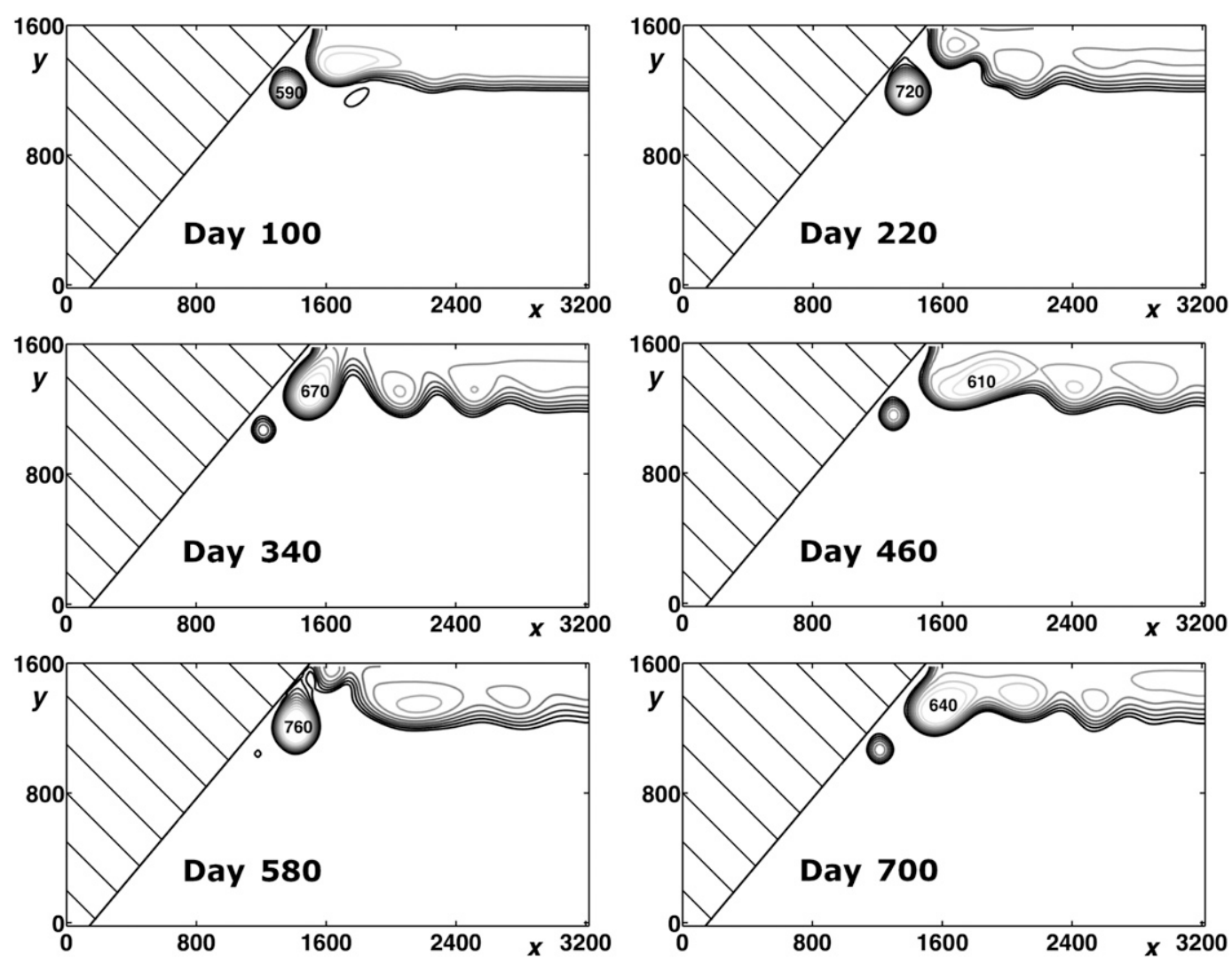

FIG. 8. The numerical simulations for the $\mathrm{BC}\left(\gamma=50^{\circ}\right)$. The detached rings are almost stationary and later recaptured. Such a scenario is qualitatively similar to that observed in the EAC retroflection area.

that this magnified speed leads to an increase in the eddy propagation rate and a decrease in the shedding period and the critical slant by about $10^{\circ}$. However, the theoretical magnification is compensated for in the numerics by viscosity, which dramatically reduces the speeds.) The initial thickness of the upper layer at the wall was taken to be 0 and $300 \mathrm{~m}$. The modeled time in all our experiments was long, about 700 days. The aforementioned resolution choices were adequate for our Rossby radius of $30 \mathrm{~km}$, which corresponded to $H \approx 350 \mathrm{~m}$ (with our choice of $g^{\prime}$ and $f_{0}$ ). Furthermore, these choices always allowed for at least nine grid points across the downstream current, which is also adequate.

We note that the resulting upper-layer thickness contours are qualitatively similar to each other for the various runs. Figure 8 shows the maps for $h_{0}=0 \mathrm{~m}$ and an initial $\alpha$ of unity. The plots are shown for each 120 days, from $t=100$ to $t=700$ days. The scales in $x$ and $y$ directions are given in kilometers. The contours of upperlayer thickness are given through $50 \mathrm{~m}$ (starting from $200 \mathrm{~m}$ ) and maximal thicknesses are also given in meters inside the closed contours. We see that, although the rings are sometimes detached from the retroflection area, they weaken quickly and are either recaptured by the following ring or disappear owing to weakening. As a result, contours for day 340 and day 700 are almost the same near the coast, with one small eddy that is recaptured later.

We should note the difference between Fig. 8, in this paper, and Fig. 7 in $\mathrm{ZNb}$ : the weakening of rings is significantly stronger here. This is probably caused by the viscosity that is nominally the same but is relatively stronger here because $Q$ is $20 \mathrm{~Sv}$ instead of $70 \mathrm{~Sv}$ in the Agulhas case. Regardless, we suggest that, in the real ocean, most of the rings that would have temporarily detached from the $\mathrm{BC}$ in the absence of $\mathrm{MC}$ (at the point of its retroflection) would have been recaptured by the BC. For a comparison, Fig. 9 shows the contours for the same BC parameters but the hypothetical case of $\gamma=25^{\circ}$ instead of the real $50^{\circ}$; that is, the wall joins the points $(x=0, y=360 \mathrm{~km})$ and $(x=2600, y=1600 \mathrm{~km})$, and initially the current retroflects at about $y=930 \mathrm{~km}$. It is seen that, in this case, although the capturing occurs sometimes, the chain of eddies does form gradually, and almost four complete eddies could be seen for the 700th day. This is the case for subcritical coastal slant. 

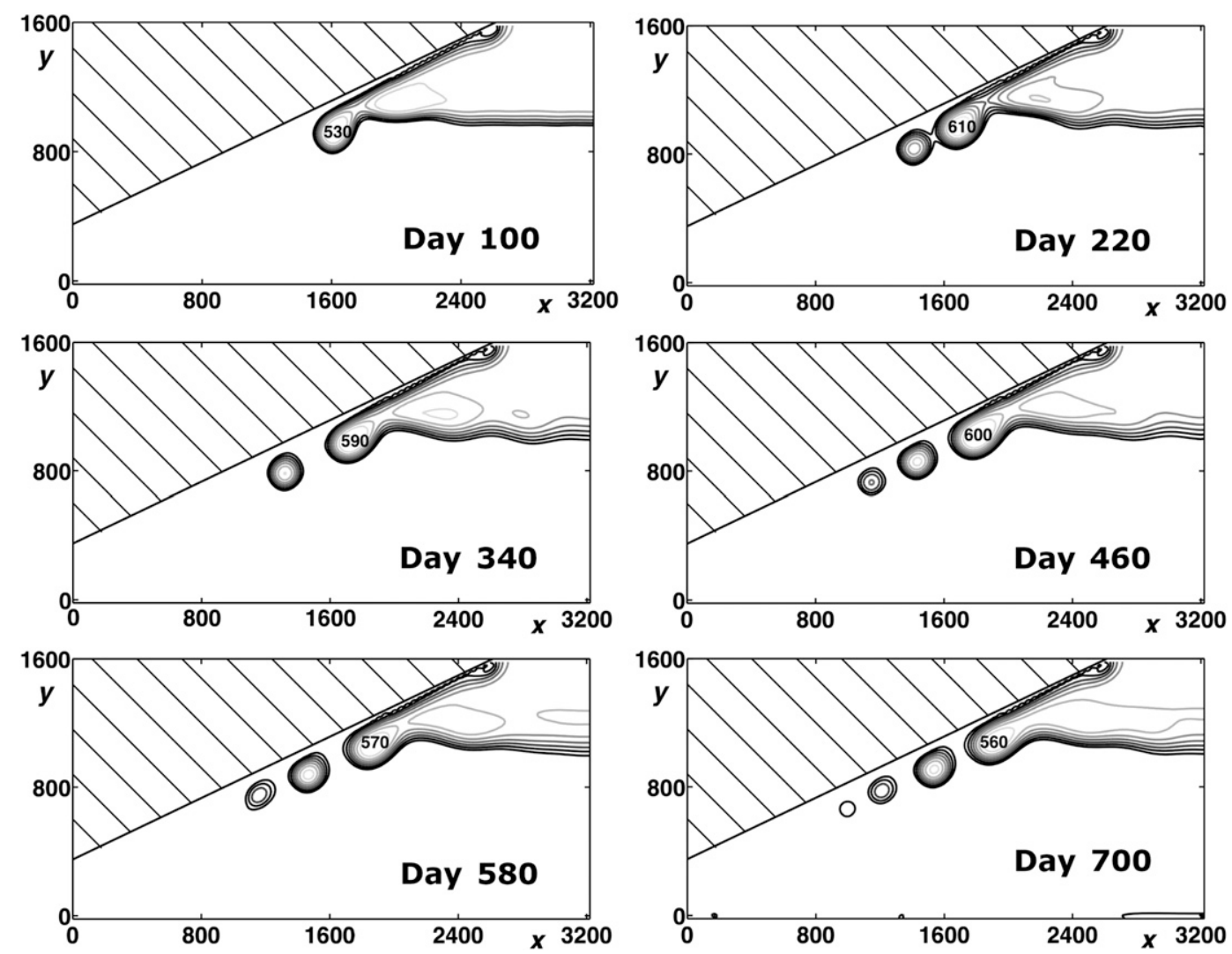

FIG. 9. Numerical simulations for the hypothetical BC with a coastal slant of $25^{\circ}$ (which is subcritical) instead of the actual angle of $50^{\circ}$. Note that a chain of eddies appears.

For the NBC, we took $f_{0}=2.03 \times 10^{-5} \mathrm{~s}^{-1}, Q=35 \mathrm{~Sv}$, $g^{\prime}=2 \times 10^{-2} \mathrm{~m} \mathrm{~s}^{-2}$, and $\beta=6 \times 10^{-11} \mathrm{~m}^{-1} \mathrm{~s}^{-1}$. Since the NBC is in the Northern Hemisphere, we took a basin of the same size as for the $\mathrm{BC}$, but the coast was represented by a line joining the points $(x=0 \mathrm{~km}, y=280 \mathrm{~km})$ and $(x=3200 \mathrm{~km}, y=0)$; implying $\gamma=5^{\circ}$. For the initial point of retroflection, we took $x=1940 \mathrm{~km}$. The contours for $h_{0}=0$ and an initial $\alpha$ of unity are shown in Fig. 10 . The plots here are given for every 40 days, from $t=40$ to $t=240$ days, and the scales are the same as in Figs. 8 and 9: we were obliged to take $\nu=1010 \mathrm{~m}^{2} \mathrm{~s}^{-1}$ so as to ensure the stability of the calculations. Consequently, the runs were subjected to a very strong effect of viscosity, resulting in a quick weakening of the rings chain after the first one was formed. Nevertheless, the plots do confirm that the considered coastline slant is subcritical because the first eddy passed the left boundary of the plot frame merely after 200 days of simulation, and the second approaches it after 240 days. In addition, a chain of at least three eddies is clearly seen for the 160th and 200th days. The third and fourth eddies are propagating with the same rate as the first and second. They are gradually damped by viscosity but, as stated, are not recaptured.

\section{Mass fluxes}

The difference in shedding regimes can also be confirmed by the relationship between the incoming volume flux $Q$ and the outgoing flux $q$. When the shedding is weak, $q$ and $Q$ are approximately equal $(\Phi \approx 0)$, whereas strong shedding implies $\Phi \gg 0$ [where $\Phi=(Q-q) / Q$ ]. Using a least squares fit we found for the BC (with no MC) that $\Phi=0$ when $\alpha<0.114$ and $\Phi=0.021 \alpha^{2}+$ $0.446 \alpha-0.051$ otherwise, implying that, when $\alpha$ is approximately 0.1 (adequate for the BC), $\Phi$ is almost zero; that is, the current retroflects completely, and no mass flux goes into eddies. For the NBC, on the other hand, we obtained $\Phi=0$ when $\alpha<0.138$ and $\Phi=-0.657 \alpha^{2}+$ $1.663 \alpha-0.217$ otherwise. This implies that, although $\Phi$ is zero for a broader range of $\alpha$, it is greater than that for the $\mathrm{BC}$ for all values of $\alpha$ exceeding 0.15. As mentioned above, for the NBC $\alpha$ is about 0.5 , giving $\Phi \approx 0.45$, implying that a little more than one-half of the NBC retroflects ( $q$ is $20 \mathrm{~Sv}$ when $Q$ is $35 \mathrm{~Sv}$ ).

Figure 11 shows the incoming and outgoing fluxes for the BC (upper panel) and NBC (lower panel) versus time. We did not smooth out the high-frequency noise 

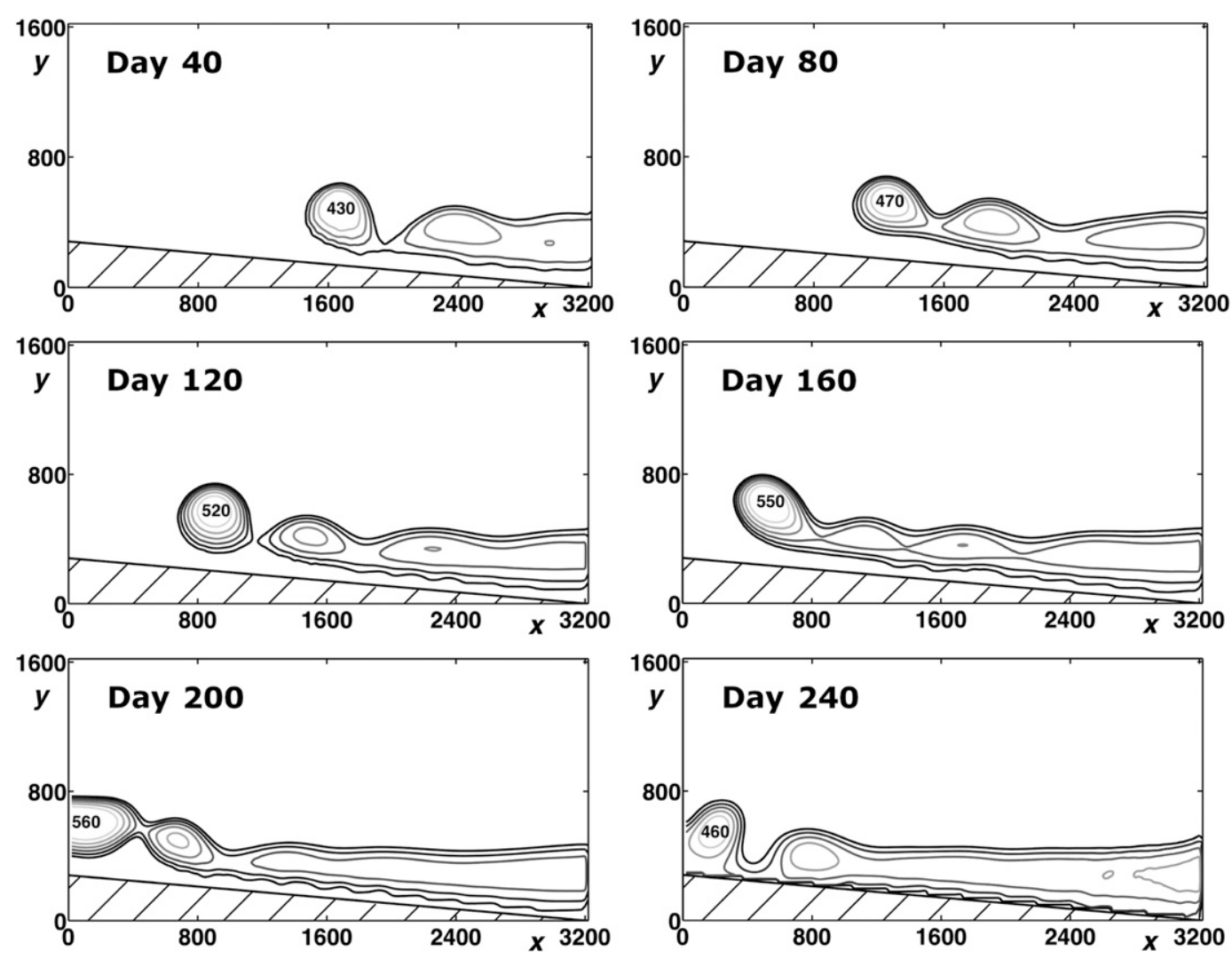

FIG. 10. Numerical simulations of the NBC retroflection. A chain of eddies is seen here despite the strong effect of the (unavoidable) high viscosity.

(as we did in $\mathrm{ZNa}$ ), and it could be as much as 3-4 (Sv). Unfortunately, at the end of the calculations of the second case (for 240th day), the stability of computations worsens dramatically, and further analysis is invalid. It is seen that, for the $\mathrm{BC}$, almost all incoming flux retroflects and, because of the noise, $q$ sometimes even exceeds $Q$. For the NBC, on the other hand, our theoretical prediction of $q$ (about $20 \mathrm{~Sv}$ ) is more accurately confirmed (bearing in mind that, owing to viscosity, $\alpha$ gradually decreases during the numerical run, leading to an increase in $q$ ).

\section{Summary and discussion}

Both theory and numerical simulations of the $\mathrm{BC}$ and the NBC show that the coastal slant is subcritical for the NBC but supercritical for the BC (Figs. 6-10). According to our critical coastal slant idea, suggested originally in $\mathrm{ZNb}$ for the Agulhas Current, the rings shed from the retroflection area of the NBC are expected to propagate along the coast away from the retroflection, forming a relatively stable chain, just as the Agulhas eddies do. $\mathrm{By}$ contrast, the $\mathrm{BC}$ rings are expected to behave simi- larly to the East Australian Current (EAC) rings. [for details on EAC rings, see, e.g., Nilsson and Cresswell (1981); Sokolov and Rintoul (2000). The coastline slant near the EAC retroflection is about $65^{\circ}$, which is supercritical for each value of $\alpha$.] Namely, the rings detach from the retroflection area at a slow rate and, in most cases, dissipate without forming a chain or are recaptured by the incipient eddies or meanders of the retroflected flow behind.

Figure 8 shows that, because of the large coastal slant, the $\mathrm{BC}$ would have not generated eddies even if there would have not been a Malvinas Current. On the other hand, Fig. 9, which is very illuminating, illustrates that, just like the NBC, the $\mathrm{BC}$ would have generated rings had there been no Malvinas and the coastline slant would have been smaller. This suggests that the occasionally observed rings that are shed from the $\mathrm{BC}$ are probably caused by the $\mathrm{BC}$ collision with the relatively strong MC (see, e.g., Vivier and Provost 1999, Olson et al.1988) rather than by the $\mathrm{BC}$ retroflection itself. The detail consideration of such a process is left for future investigations, but we speculate here that the MC advects rings away from their generation area so that they 

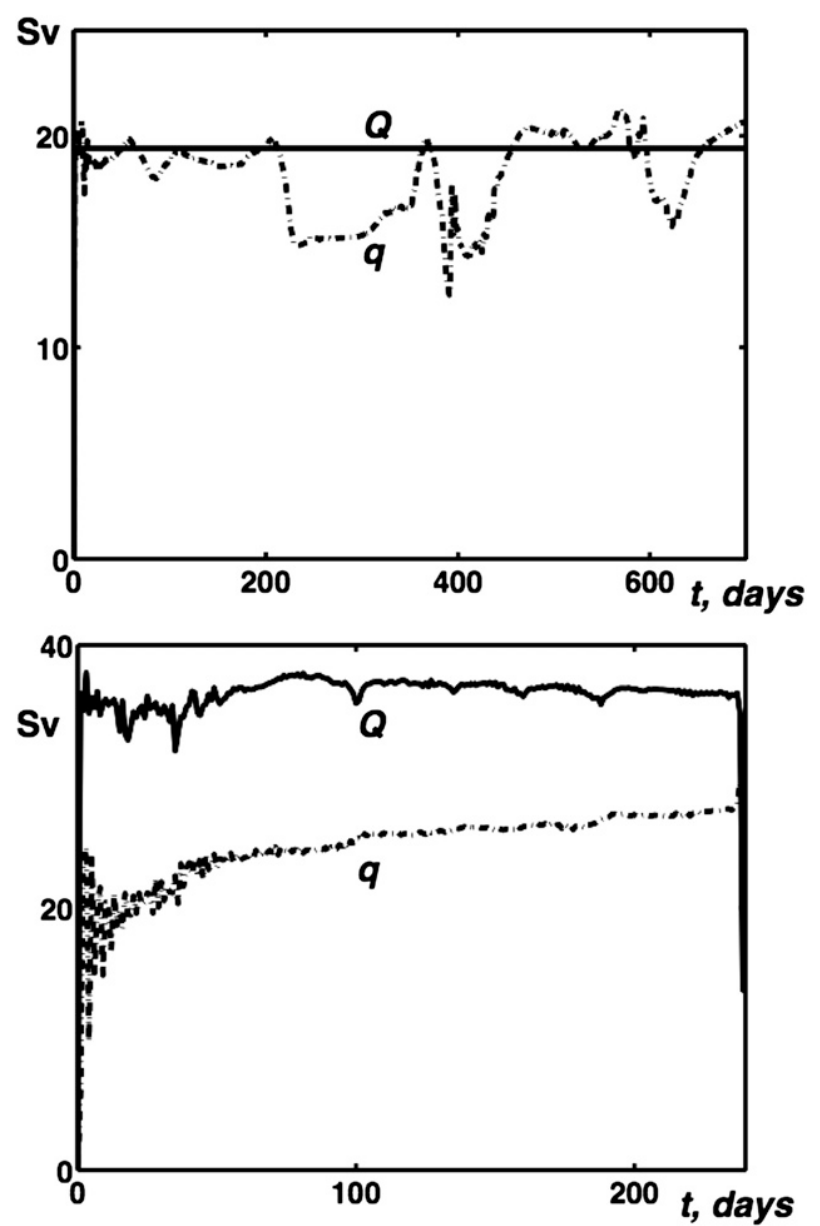

FIG. 11. Incoming $Q$ and outgoing $q$ mass fluxes (top) for the BC calculated for the same conditions as those of Fig. 8 and (bottom) for the NBC for the same conditions as those of Fig. 10 vs time. Note that, in the second case, a much smaller fraction of the incoming flux retroflects.

are not recaptured. Hence, the role of the MC in ring generation is probably very different from that which was previously thought. Paradoxically, instead of preventing rings generation (by providing an opposing flow force) in the first place, it actually helps generate rings by removing them from the formation area before they are recaptured by the following current.

Note that, owing to the proximity of the NBC to the equator $\left(8^{\circ} \mathrm{N}\right)$, our assumptions of a small $\varepsilon$ and circular rings may give significant errors. Furthermore, our approach is completely invalided when the theoretical radius of eddy exceeds the distance between its center and the equator. Given that $R \sim 2^{2 / 3} R_{d}$ (see, e.g., Nof and Pichevin 2001), the Coriolis parameter near the equator is $\beta y$, this validity limit is $8 g^{\prime} H(\beta y)^{-2} \sim y^{2}$, giving

$$
y \sim\left(\frac{8 g^{\prime} H}{\beta^{2}}\right)^{1 / 4} .
$$

For $g^{\prime}=0.02 \mathrm{~m} \mathrm{~s}^{-2}$ and $H=700 \mathrm{~m}$, this is $6.1^{\circ}$ of latitude, which is fairly close to our $8^{\circ} \mathrm{N}$ choice for the NBC. With this choice, all slanted coastlines to the east of Surinam are included in this experiment. The associated errors are leading to significantly larger radii in the southern parts of eddies than in the northern parts. With a significant $\beta$, the associated particles trajectories become trochoidal rather than circular. Therefore, eddies that may have leaked on their southern side could have led to the strong blurriness seen in Fig. 10.

We note that the decrease in the calculated critical slant angle when approaching the equator is due to the sharp increase of the BE propagation rate [with our approach (2.21), the westward speed is inversely proportional to the square of the Coriolis parameter.] In real conditions, this velocity does not increase that sharply because of friction, which increases with the square of the velocity. For example, the theoretical propagation rate of eddies shed by the NBC is about 10 times the Agulhas rings propagation rate, even though the slant does not differ that much from that of the NBC. In the ocean, however, the average propagation rate of the NBC is merely about 2-2.5 times that of Agulhas rings (see, e.g., Lutjeharms 2006 for Agulhas Current; Johns et al. 1990 and Goni and Johns 2001 for NBC rings). Therefore, we expect that the actual critical slant will not decrease as strongly when approaching the equator, and this is in agreement with our numerics. In the area where our theoretical model does not work very well because of the smallness of the Coriolis parameter, we theoretically obtain very small critical angles, much less than $25^{\circ}-30^{\circ}$. Hence, the averaged slant of the entire coast of northern South America is all within the subcritical range. This is why eddy shedding occurs constantly despite the seasonal migration of the NBC retroflection.

Finally, we note that it is very difficult to verify the current and eddy volume partitioning $(\Phi)$ in field experiments because of opposing current convergences [Brazil-Malvinas and retroflected NBC-North Equatorial Current (NEC)]. This is particularly true with the retroflected southeast flow of the NBC that forms the North Equatorial Countercurrent (NECC) together with the retroflected NEC exceeding the incoming flux of the NBC by as much as 7 Sv (Garzoli et al. 2004).

Acknowledgments. This study was supported by the following grants: a NASA doctoral fellowship (NNG05GP65H), the NSF (OCE-0752225, OCE-9911342, OCE-0545204 and OCE-0241036), LANL/IGPP (1815), BSF (2006296), and NASA (NNX07AL97G). Volodymyr Zharkov was also supported by the Jim and Sheila O'Brien Fellowship. 


\section{APPENDIX}

\section{List of Symbols and Abbreviations}

$C_{x o}$

eddy drift velocity in the open ocean

$C_{x}$

$C_{y}$

$C_{\xi}, C_{\eta}$

$C_{\xi f}$

$C_{\xi l}, C_{\xi \xi}$

eddy migration rate in the zonal direction

eddy migration rate in the meridional direction

eddy migration rates in the tilted coordinate

system

eddy migration rate along the slanted coast

after detachment

respectively

$d$

$d_{u}$

$d_{1}$

$d_{2}$

$F_{1}$

$F_{2}$

$F_{3}$

$F_{4}$

$f$

$f_{0}$

$g^{\prime}$

$h$

$H$

$H_{f l}, H_{f u}$

$h_{0}$

$\tilde{h}$

$\bar{h}$

$Q$

$q$

$R$

$R_{d}$

$R_{f}$

$R_{i}$

$R_{f}, R_{f u}$

$r$

$s$

$t$

$\hat{t}$

$t_{f}$

$t_{f}, t_{f u}$

$u, \nu$

$u^{*}, \nu^{*}$

$u_{1}$ distance between two consecutive eddies

"upper" boundary of $d$

width of incoming current

width of retroflected current

force of incoming current

force of retroflected current

Coriolis force associated with the migration of the eddy

\section{$\beta$ force of the eddy}

the Coriolis parameter

approximate Coriolis parameter at the eddy center

reduced gravity

upper-layer thickness

upper-layer thickness outside the retroflection area

thicknesses at the moments $t_{f l}, t_{f u}$, respectively upper-layer thickness at the wall

upper-layer thickness in the stagnant wedge situated between the incoming and retroflected currents

undisturbed upper-layer thickness

mass flux of the incoming current

mass flux of the retroflected current

radius of the eddy (a function of time)

Rossby radius of the eddy (a function of time)

final radius of the eddy at the moment of detachment

initial radius of the eddy

"lower" and "upper" boundaries of $R_{f}$

radius in polar coordinate system

area of integration

time

time in the fixed coordinate system

period of eddies' generation

"lower" and "upper" boundaries of $t_{f}$

velocities in the zonal and meridional directions

velocities in the tilted coordinate system axes

absolute value of the current velocity at the edge

V

$\overline{v_{\theta}}$

$x, y$

$\alpha$

$\beta$

$\gamma$

$\delta_{1}, \delta_{2}$

$\Delta \rho$

$\varepsilon$

$\theta$

$\mu$

$\nu$

$\xi, \eta$

$\rho$

$\Phi$

$\phi$

$\psi$ volume of the eddy

orbital speed

zonal and meridional coordinate axes in the moving system

vorticity (twice the Rossby number)

meridional gradient of the Coriolis parameter

coastline slant

differences between the eddy radius and current widths $d_{1}, d_{2}$, respectively

difference between densities of lower and upper layer

small parameter defined as $\beta R_{d} / f_{0}$

angle in polar coordinate system

parameter defined as $2 \pi R d R / d t$

viscosity coefficient (in numerics)

axes of tilted moving coordinate system

upper-layer density

part of current's mass flux going into eddies

path of integration

streamfunction

\section{REFERENCES}

Bischof, B., A. J. Mariano, and E. H. Ryan, cited 2003: The North Brazil Current. [Available online at http://oceancurrents. rsmas.miami.edu/atlantic/north-brazil.html.]

Bleck, R., and D. Boudra, 1986: Wind-driven spin-up in eddyresolving ocean models formulated in isopycnic and isobaric coordinates. J. Geophys. Res., 91 (C6), 7611-7621.

Bourles, B., Y. Gouriou, and R. Chuchla, 1999: On the circulation and upper layer of the western equatorial Atlantic. J. Geophys. Res., 104 (C9), 21 151-21 170.

da Silveira, I. C. A., L. B. Miranda, and W. S. Brown, 1994: On the origins of the North Brazil Current. J. Geophys. Res., 99 (C11), 22 501-22 512.

_ - G. R. Flierl, and W. S. Brown, 1999: Dynamics of separating western boundary currents. J. Phys. Oceanogr., 29, 119-144.

Fratantoni, D. M., and D. A. Glickson, 2002: North Brazil Current ring generation and evolution observed with SeaWiFS. J. Phys. Oceanogr., 32, 1058-1074.

Garzoli, S. L., A. Ffield, W. E. Johns, and Q. Yao, 2004: North Brazil Current retroflection and transports. J. Geophys. Res., 109, C1013, doi:10.1029/2003JC001775.

Goni, G. J., and W. E. Johns, 2001: A census of North Brazil Current rings observed from TOPEX/POSEIDON altimetry: 1992-1998. Geophys. Res. Lett., 28, 1-4.

Jochum, M., and P. Malanotte-Rizzoli, 2003: On the generation of North Brazil Current Rings. J. Mar. Res., 61, 147-173.

Johns, W. E., T. N. Lee, F. A. Schott, R. J. Zantopp, and R. H. Evans, 1990: The North Brazil Current retroflection: Seasonal structure and eddy variability. J. Geophys. Res., 95 (C12), 22 103-22 120.

,-- , R. C. Beardsley, J. Candela, R. Limeburner, and B. Castro, 1998: Annual cycle and variability of the North Brazil Current. J. Phys. Oceanogr., 28, 103-128.

Lentini, C. A. D., G. G. Podesta, E. J. D. Campos, and D. B. Olson, 2001: Sea surface temperature anomalies on the Western South Atlantic from 1982-1994. Cont. Shelf Res., 21, 89-112. 
Lumpkin, R., and S. L. Garzoli, 2005: Near-surface circulation in the Tropical Atlantic Ocean. Deep-Sea Res. I, 52, 495-518.

Lutjeharms, J. R. E., 2006: The Agulhas Current. Springer-Verlag, $330 \mathrm{pp}$.

Nilsson, C. S., and G. R. Cresswell, 1981: The formation and evolution of East Australian Current warm-core eddies. Prog. Oceanogr., 9, 133-183.

Nof, D., 1983: On the migration of isolated eddies with application to Gulf Stream rings. J. Mar. Res., 41, 399-425.

— 1996: Why are rings regularly shed in the western equatorial Atlantic but not in the western Pacific? Prog. Oceanogr., 38, 417-451.

_ 1999: Strange encounters of eddies with walls. J. Mar. Res., 57, 1-24.

— of anticyclonic eddies. J. Phys. Oceanogr., 17, 343-357.

— , and T. Pichevin, 2001: The ballooning of outflows. J. Phys. Oceanogr., 31, 3045-3058.

Olson, D. B., G. P. Podesta, R. H. Evans, and O. Brown, 1988: Temporal variations in the separation of the Brazil and Malvinas currents. Deep-Sea Res., 35A, 1971-1990.

Orlanski, I., 1976: A simple boundary condition for unbounded hyperbolic flows. J. Comput. Phys., 21, 251-269.

Palma, E. D., R. P. Matano, and A. R. Piola, 2008: A numerical study of the Southwestern Atlantic Shelf circulation: Stratified ocean response to local and offshore forcing. J. Geophys. Res., 113, C11010, doi:10.1029/2007JC004720.

Peterson, R. G., and L. Stramma, 1991: Upper-level circulation in the South Atlantic Ocean. Prog. Oceanogr., 26, 1-73.

Pichevin, T., and D. Nof, 1996: The eddy cannon. Deep-Sea Res. I, 43, 1475-1507.

_, — , and J. R. E. Lutjeharms, 1999: Why are there Agulhas rings? J. Phys. Oceanogr., 29, 693-707.

Robinson, A. R., 1983: Eddies in Marine Science. Springer-Verlag, 609 pp.

Schott, F. A., J. Fischer, and L. Stramma, 1998: Transports and pathways of the upper-layer circulation in the western tropical Atlantic. J. Phys. Oceanogr., 28, 1904-1928.

Sokolov, S., and S. Rintoul, 2000: Circulation and water masses of the southwest Pacific: WOCE Section P11, Papua New Guinea to Tasmania. J. Mar. Res., 58, 223-268.

Vivier, F., and C. Provost, 1999: Direct velocity measurements in the Malvinas Current. J. Geophys. Res., 104 (C9), $21083-$ 21103.

Zharkov, V., and D. Nof, 2008a: Retroflection from slanted coastlines - Circumventing the "vorticity paradox." Ocean Sci., 4, 293-306.

- , and - 2008b: Modeling of Agulhas ring injection into the South Atlantic during glacials and interglacials. Ocean Sci., 4, 223-237. 